\title{
A New Nonlocal Maximum Likelihood Estimation Method for Denoising Magnetic Resonance Images
}

\author{
Jeny Rajan ${ }^{1,2}$, Arnold J. den Dekker ${ }^{3}$, Jaber Juntu ${ }^{2}$, and Jan Sijbers ${ }^{2}$ \\ 1 Department of Computer Science and Engineering, National Institute of \\ Technology - Karnataka, Surathkal, India \\ 2 iMinds Vision Lab, Department of Physics, University of Antwerp, Belgium \\ 3 Delft Center for Systems and Control, Delft University of Technology, 2628 CD \\ Delft, The Netherlands
}

\begin{abstract}
Denoising of Magnetic Resonance images is important for proper visual analysis, accurate parameter estimation, and for further preprocessing of these images. Maximum Likelihood (ML) estimation methods were proved to be very effective in denoising Magnetic Resonance (MR) images. Among the ML based methods, the recently proposed Non Local Maximum Likelihood (NLML) approach gained much attention. In the NLML method, the samples for the ML estimation of the true underlying intensity are selected in a non local way based on the intensity similarity of the pixel neighborhoods. This similarity is generally measured using the Euclidean distance. A drawback of this approach is the usage of a fixed sample size for the ML estimation and, as a result, optimal results cannot be achieved because of over- or under-smoothing. In this work, we propose an NLML estimation method for denoising MR images in which the samples are selected in an adaptive way using the Kolmogorov-Smirnov (KS) similarity test. The method has been tested both on simulated and real data, showing its effectiveness.
\end{abstract}

Keywords: Image denoising, Kolmogorov-Smirnov test, MRI, Noise, Rice distribution.

\section{Introduction}

Denoising algorithms play an important role in the enhancement of MR images. Noise in MRI can be naturally minimized by averaging images after multiple acquisitions. This, however, may not be feasible in clinical and small animal MR imaging where there is an increasing need for speed [7. Thus, post processing techniques to remove noise in the acquired data are important. Also, in time-sensitive acquisitions or studies with limited imaging time (diffusion MRI, functional MRI etc.), experiments cannot be repeated to do averaging. In the early days, many authors applied the conventional classical denoising techniques to denoise MRI [5. These methods assumed the noise in the image to be Gaussian distributed. The major drawback of these methods is that the biasing effects 
of Rician noise, which characterizes magnitude MR images, were not taken into account. This bias increases with decreasing SNR. Later many methods were proposed to denoise MR images. Most of these methods exploited the second raw moment of the Rice distribution to reduce the bias in the denoised images [78]. That is, the image is denoised with the methods based on the Gaussian assumption and to reduce the bias, $2 \sigma_{-} g^{2}$ is subtracted from the squared denoised image (where $\sigma_{-} g^{2}$ is the variance of the noise in the complex image). However, in [14] it is shown that the sample size and SNR have a significant influence on the process of estimating the true underlying signal using this approach. ML methods were proved to be better than the aforementioned methods 9$]$.

ML based denoising methods applied to magnitude MR images incorporate the Rice distribution to estimate the true underlying signal from a local neighborhood within which the signal is assumed to be constant. However, if this assumption is not met, such filtering will lead to blurred edges and loss of fine structures in the image. As a solution to the blurring issue of the local ML approach, the non local ML (NLML) estimation method [6] was proposed. In the NLML method, the samples for the ML estimation of the true underlying intensity are selected in a non local (NL) way based on the intensity similarity of the pixel neighborhoods. This similarity is generally measured using the Euclidean distance [6]. In that method, however, the number of NL pixels to be considered for ML estimation is fixed and is determined in a heuristic way. This fixed sample size can introduce under- or over-smoothing in the images. In this work, we propose a non local ML estimation method for denoising MR images in which the samples are selected in an adaptive way using the Kolmogorov-Smirnov similarity test.

This paper is organized as follows. Section 2 gives a short overview about the noise characteristics in MR images. In Section 3, the signal estimation using conventional NLML is discussed. Section 4 elaborates the proposed method. Section 5 presents the experimental results, comparative evaluation and discussion. Finally, conclusions and remarks are drawn in Section 6.

\section{Noise Distribution in MR Images}

The acquired complex valued MR signals in the $k$-space are characterized by a zero mean Gaussian probability density function (PDF). The $k$-space data are then (inverse) Fourier transformed to obtain the magnetization distribution. After the inverse Fourier transform, the noise distribution in the real and imaginary components will still be Gaussian distributed due to the linearity and the orthogonality of the Fourier transform. However, due to the subsequent transform to a magnitude image, the data will no longer be Gaussian but Rician distributed.

Let $R$ and $I$ represent the real and imaginary parts of the noisy complex MR data (corrupted by zero mean Gaussian, stationary noise with the standard deviation $\sigma_{g}$ ) with mean values $\mu_{R}$ and $\mu_{I}$, respectively. Then the PDF of the reconstructed magnitude data $M$ will be [12] 


$$
p_{\mathrm{M}}\left(M \mid A, \sigma_{g}\right)=\frac{M}{\sigma_{g}^{2}} e^{-\frac{M^{2}+A^{2}}{2 \sigma_{g}^{2}}} I_{0}\left(\frac{A M}{\sigma_{g}^{2}}\right) \epsilon(M)
$$

where $M=\sqrt{R^{2}+I^{2}}, A=\sqrt{\mu_{R}^{2}+\mu_{I}^{2}}, I_{0}($.$) is the 0^{t h}$ order modified Bessel function of the first kind and $\epsilon($.$) is the Heaviside step function.$

Proper estimation of the noise variance, $\sigma_{g}^{2}$, is important for effective denoising of MR images. Noise can be estimated from an MR image in a number of ways 15 16 11110. A survey of these methods is given in 2]. In this work, we used the object based methods proposed in [10] for the estimation of noise.

\section{Signal Estimation Using NLML Method}

Let $M_{1}, M_{2}, \ldots, M_{n}$ be $n$ statistically independent observations within a region of constant signal intensity $A$. Then, the joint PDF of the observations is:

$$
p\left(\left\{M_{i}\right\} \mid A, \sigma_{g}\right)=\prod_{i=1}^{n} \frac{M_{i}}{\sigma_{g}^{2}} e^{-\frac{M_{i}^{2}+A^{2}}{2 \sigma_{g}^{2}}} I_{0}\left(\frac{A M_{i}}{\sigma_{g}^{2}}\right)
$$

The ML estimator of $A$ can now be computed by maximizing the likelihood function $L(A)$ or equivalently $\ln L(A)$, with respect to $A$ [13]:

$$
\ln L=\sum_{i=1}^{n} \ln \left(\frac{M_{i}}{\sigma_{g}^{2}}\right)-\sum_{i=1}^{n} \frac{M_{i}^{2}+A^{2}}{2 \sigma_{g}^{2}}+\sum_{i=1}^{n} \ln I_{0}\left(\frac{A M_{i}}{\sigma_{g}^{2}}\right)
$$

and

$$
\widehat{A}_{\mathrm{ML}}=\arg \max _{A}(\ln L)
$$

Nevertheless, to estimate the true underlying intensity $\widehat{A}_{M L}$ for each pixel $i$ in the image using Eq. (4), samples $\left\{M_{i}\right\}$ with similar underlying intensity need to be selected. The straightforward approach to select samples $\left\{M_{i}\right\}$ is to select all pixels from a local neighborhood of $i$. However, it is clear that around edges and fine structures the assumption of uniform underlying intensity is violated, and, as a result, blurring will be introduced in the image. An alternate approach is to use non local (NL) pixels instead [6. Conventionally, NL pixels are selected based on the intensity similarity of the pixel neighborhoods. If the neighborhoods of two pixels are similar, then their central pixels should have a similar meaning and thus similar gray values [19]. The similarity of the pixel neighborhoods can be computed by taking the intensity distance (Euclidian distance) between them 6]:

$$
d_{i, j}=\left\|N_{i}-N_{j}\right\|
$$

where $d_{i, j}$ is the intensity distance between the neighborhoods $N_{i}$ and $N_{j}$ of the pixels $i$ and $j$. For each pixel $i$, the intensity distance $d$ between $i$ and all other non local pixels $j$ as defined by Eq. (5), in the search window are measured. The first $k$ pixels are then selected as $\left\{M_{i}\right\}$ after sorting the NL pixels in the 
increasing order of the distance $d$ for the maximum likelihood (ML) estimation. This method is more effective in terms of preserving edges and fine structures than the local ML estimation. However, one major concern with this approach is the proper selection of $k$. In the conventional NLML method $[\underline{6}, k$ is fixed and generally determined in a heuristic way. Low or high values of $k$ can cause under or over smoothing. In the section below, we discuss an alternative approach to select the samples for ML estimation.

\section{NLML Method Based on Kolmogorov-Smirnov (KS) Similarity Test}

The research presented in this section is motivated by the study in [18. In [18, it is observed that the PDF of an image $S$, resulting from the difference of two images $R_{1}$ and $R_{2}(S=R 1-R 2)$ with the similar underlying intensity and corrupted with Rician noise, is symmetric and can be approximated with a Gaussian distribution of mean $\mu_{s}$ and standard deviation $\sigma_{s}$ given by

$$
\mu_{s}=0, \quad \sigma_{s}=\sqrt{2} \sigma_{r}
$$

where $\sigma_{r}$ is the standard deviation of the Rice distribution. When the SNR increases, $\sigma_{s}$ approaches $\sqrt{2} \sigma_{g}$ since $\sigma_{r}$ approaches $\sigma_{g}$ for high SNR.

In this work, we make use of the above knowledge, instead of using the Euclidian distance, to find the samples for estimating the true underlying intensity for each pixel in the image. For every pixel $i$, difference patches $D_{i, j}$ with other NL pixels $j$, based on the neighborhoods of $i$ and $j$, are first created. If the neighborhoods $N_{i}$ and $N_{j}$ of the pixels $i$ and $j$ are similar, then the distribution of the difference patch $D_{i, j}$ can be approximated with a Gaussian distribution with the parameter values as given in Eq. (6).

To test the normality of the distribution of $D$, the KS test was employed. The KS test was used to compare the distribution of $D$ to a standard normal distribution after standardizing $D$. The null hypothesis of the KS test is that the difference patches $D$ are normally distributed, and this hypothesis is rejected if the $p$-value of the KS test statistic is below 0.05 (i.e., the result of the KS test is 1 if the test rejects the null hypothesis at the $5 \%$ significance level, 0 otherwise) 18 . So for each pixel $i$, the NL pixels with a $p$-value $>0.05$ are selected as the samples $\left\{M_{i}\right\}$ for the ML estimation.

The parameter, $\sigma_{r}$, can be computed directly from the neighborhood of the pixel of interest if the underlying intensity of the selected neighborhood region is constant. Also, $\sigma_{r}$ will be always less than or equal to $\sigma_{g}$. The relationship between $\sigma_{r}$ and $\sigma_{g}$ is given as [10]:

$$
\sigma_{r}=\frac{\sigma_{g}}{\sqrt{\varphi}}
$$

where $\varphi$ is a correction factor depending on the SNR and is in the range $[1 ;(2-$ $\pi / 2)^{-1}$. If the selected neighborhood contains pixels from more than one region, 
then the estimation of $\sigma_{r}$ will be difficult. However, if the estimated $\sigma_{r}$ is greater than $\sigma_{g}$, we can assume that the neighborhood contains more than one region and in those cases, in Eq. (6), we replaced $\sigma_{r}$ with $\sigma_{g}$. For the estimation of $\sigma_{g}$, we used the object based method proposed in [10].

\section{$5 \quad$ Experiments and Results}

To evaluate and compare the proposed NLML $\left(\mathrm{NLML}_{K S}\right)$ with the conventional NLML method, experiments were conducted on both synthetic and real MR images. For the experiments on the synthetic data, we used the standard MR image phantom of the brain obtained from the Brainweb database [4. The phantom image was degraded with Rician noise for a wide range of noise levels and the denoising efficiency of both algorithms were evaluated based on the Peak Signal to Noise Ratio (PSNR), the mean Structural Similarity Index Matrix (SSIM) 17], Bhattacharrya coefficient (BC) [3] and the Mean Absolute Difference (MAD).

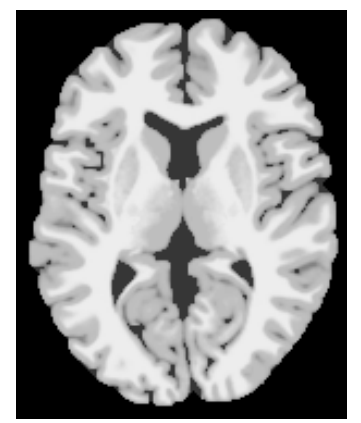

(a)

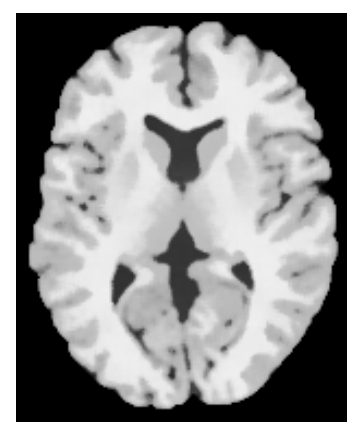

(d)

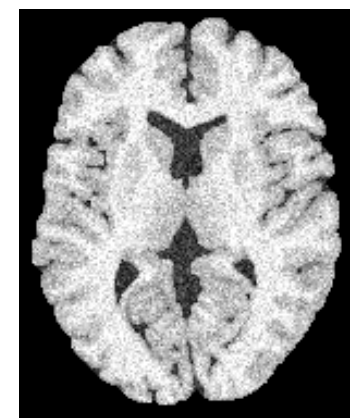

(b)

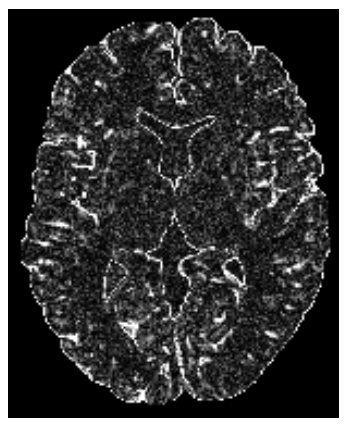

(e)

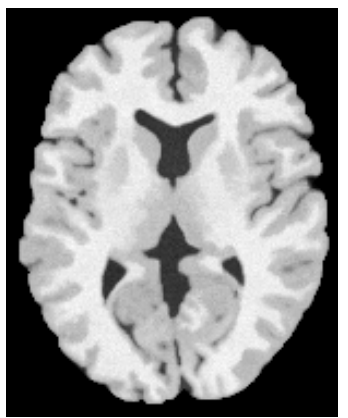

(c)

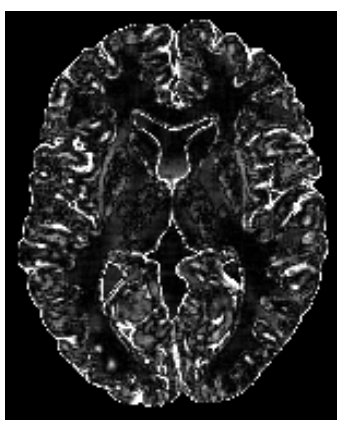

(f)

Fig. 1. Visual quality comparison: (a) Ground truth (b) Ground truth corrupted with Rician noise of $\sigma_{g}=25$ (PSNR : 21.8, MSSIM : 0.5905) (c) denoised with conventional NLML method (PSNR : 28.4, MSSIM : 0.8626)(d) denoised with the proposed method (PSNR : 28.7, MSSIM : 0.9022)(e) NLML residuals (f) NLML $K S$ residuals. The residuals are scaled in the range $0-25$. 


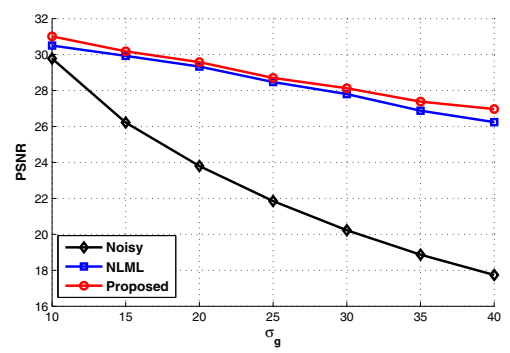

(a)

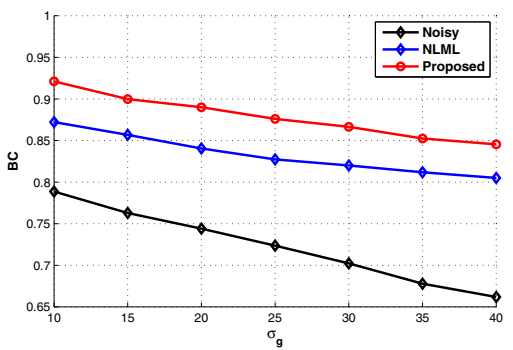

(c)

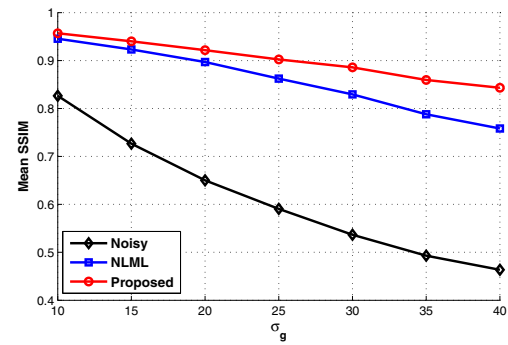

(b)

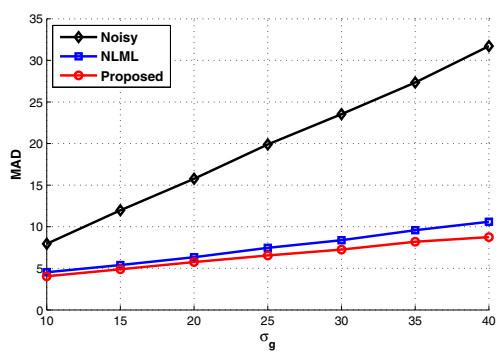

(d)

Fig. 2. Quantitative analysis of the proposed method with NLML based on (a) PSNR (b) SSIM (c) BC and (d) MAD for image corrupted with Rician noise of $\sigma_{g}$ varying from 10 to 40

Fig.1 shows the visual quality comparison of the image denoised with the NLML method and NLML $K S$ method. This experiment was conducted on the brain image after corrupting the image by noise with $\sigma_{g}=25$. It can be seen from the denoised images and from the corresponding residuals that the image denoised with the NLML $\mathrm{N}_{S}$ method is closer to the ground truth than the image denoised with the conventional NLML. Fig.2 shows the quantitative analysis of both methods in terms of PSNR, mean SSIM, BC and MAD. Both filters were executed with the following parameters: search window size $=11 \times 11 \times 5$, neighborhood size $=3 \times 3 \times 3$. For NLML, the sample size $k$ was chosen as 25 (as recommended in [6]). It can be observed from the plots that the proposed NLML $_{K S}$ performs better than the conventional NLML based on the quality matrices used for the evaluation.

For the experiments on the real data, we acquired an MR image of a cherry tomato using an experimental MRI scanner (Bruker $7 \mathrm{~T}$ scanner). The proposed NLML $_{K S}$ and the conventional NLML method were then applied on the acquired image. The results of this experiment is shown in Fig. 3. It can be seen from the images in Fig. 3 that the visual results are much better for the image denoised with the NLML $_{K S}$ in the sense that these images are less blurred than the images denoised with conventional NLML. This experiment on the real data set additionally indicates the effectiveness of the proposed method. 


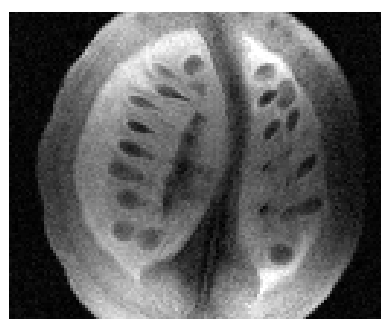

(a)

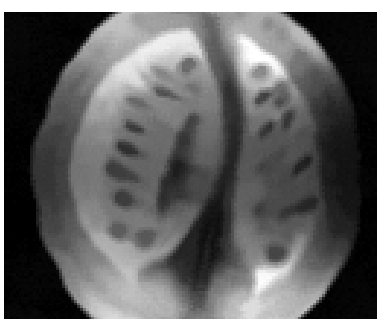

(b)

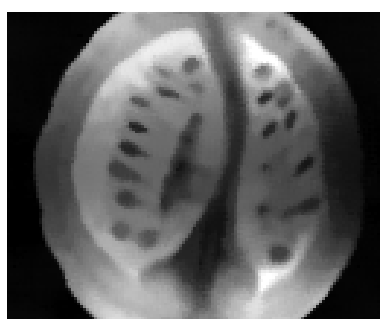

(c)

Fig. 3. Experiments on the MR image of cherry tomato. (a) Original image (b) denoised with NLML method (c) denoised with NLML $_{K S}$

\section{Conclusion}

We have proposed an NLML method based on the KS test to denoise the MR images. In the existing NLML method, the number of samples to be selected for ML estimation was based on heuristics. The proposed NLML method based on the KS test is statistically convincing and performs better (in terms of image quality) than the NLML method based on the Euclidian distance. Through the proposed KS test approach for the sample selection, the over and under smoothing caused by the conventional NLML can be reduced. Experiments have been carried out on simulated and real data sets. Quantitative analysis at various noise levels based on the similarity measures, PSNR, SSIM, BC and MAD shows that the proposed method is more effective than conventional NLML. Experiments were also performed on real MR images to prove the efficacy of the proposed method. However, it might be also possible to select the samples adaptively for the conventional NLML method based on a $\chi^{2}$-test on the squared neighborhood differences. We are also working in that direction.

Acknowledgements. This work was financially supported by the InterUniversity Attraction Poles Program of the Belgian Science Policy, and by the Fund for Scientific Research in Flanders.

\section{References}

1. Aja-Fernández, S., Alberola-López, C., Westin, C.: Noise and signal estimation in magnitude MRI and rician distributed images:a LMMSE approach. IEEE Trans. Imag. Proc. 17, 1383-1398 (2008)

2. Aja-Fernández, S., Tristán, A., Alberola-López, C.: Noise estimation in single and multiple coil magnetic resonance data based on statistical models. Magn. Reson. Imaging 27, 1397-1409 (2009)

3. Bhattacharyaa, A.: On the measure of divergence between two statistical populations defined by their probability distributions. Bull. Calcutta Math. Soc. 35, 99-109 (1943) 
4. Cocosco, C.A., Kollokian, V., Kwan, R.-S., Evans, A.C.: Brainweb: Online interface to a 3D MRI simulated brain database. NeuroImage 5(4), S425 (1997), http://www.bic.mni.mcgill.ca/brainweb/

5. Gerig, G., Kubler, O., Kikinis, R., Jolesz, F.A.: Nonlinear anisotropic filtering of MRI data. IEEE Trans. Med. Imag. 11(2), 221-232 (1992)

6. He, L., Greenshields, I.R.: A nonlocal maximum likelihood estimation method for rician noise reduction in MR images. IEEE Trans. Med. Imaging 28, 165-172 (2009)

7. Manjón, J.V., Carbonell-Caballero, J., Lull, J.J., García-Martí, G., Martí-Bonmatí, L., Robles, M.: MRI denoising using non local means. Medical Image Analysis 12, 514-523 (2008)

8. Manjón, J.V., Coupé, P., Martí-Bonmatíand, L., Collins, D.L., Robles, M.: Adaptive non local means denoising of MR images with spatially varying noise levels. J. Magn. Reson. Imaging 31, 192-203 (2010)

9. Rajan, J., Jeurissen, B., Verhoye, M., Van Audekerke, J., Sijbers, J.: Maximum likelihood estimation-based denoising of magnetic resonance images using restricted local neighborhoods. Physics in Medicine and Biology 56, 5221-5234 (2011)

10. Rajan, J., Poot, D., Juntu, J., Sijbers, J.: Noise measurement from magnitude MRI using local estimates of variance and skewness. Phys. Med. Biol. 55, N441-N449 (2010)

11. Rajan, J., Poot, D., Juntu, J., Sijbers, J.: Segmentation based noise variance estimation from background mri data. In: Campilho, A., Kamel, M. (eds.) ICIAR 2010, Part I. LNCS, vol. 6111, pp. 62-70. Springer, Heidelberg (2010)

12. Rice, S.O.: Mathematical analysis of random noise. Bell. Syst. Tech. 23, 282-332 (1944)

13. Sijbers, J., den Dekker, A.J.: Maximum likelihood estimation of signal amplitude and noise variance from MR data. Magn. Reson. Med. 51(3), 586-594 (2004)

14. Sijbers, J., den Dekker, A.J., Scheunders, P., Van Dyck, D.: Maximum likelihood estimation of Rician distribution parameters. IEEE Trans. Med. Imag. 17(3), 357361 (1998)

15. Sijbers, J., den Dekker, A.J., Verhoye, M., Van Audekerke, J., Van Dyck, D.: Estimation of noise from magnitude MR images. Magn. Reson. Imaging 16(1), 87-90 (1998)

16. Sijbers, J., Poot, D., den Dekker, A.J., Pintjens, W.: Automatic estimation of the noise variance from the histogram of a magnetic resonance image. Phys. Med. Biol. 52, 1335-1348 (2007)

17. Wang, Z., Bovik, A., Sheik, H.R., Simoncelli, E.P.: Image quality assessment: From error visibility to structural similarity. IEEE Trans. on Image Pocessing 13, 600-612 (2004)

18. Wink, A.M., Roerdink, B.T.M.: BOLD noise assumptions in fMRI. International Journal of Biomedical Imaging 2006, 1-11 (2006)

19. Zimmer, S., Didas, S., Weickert, J.: A rotationally invariant block matching strategy improving image denoising with non-local means. In: International Workshop on Local and Non-Local Approximation in Image Processing, Switzerland, pp. 135-142 (2008) 